\title{
Can Multiple Realisation be Explained?
}

\author{
Alexander Franklin* \\ August 2020 \\ Forthcoming in Philosophy

\begin{abstract}
Multiple realisation prompts the question: how is it that multiple systems all exhibit the same phenomena despite their different underlying properties? In this paper I develop a framework for addressing that question and argue that multiple realisation can be reductively explained. I illustrate this position by applying the framework to a simple example - the multiple realisation of electrical conductivity. I defend my account by addressing potential objections: contra (e.g.) (Polger and Shapiro, 2016), (Batterman, 2018), and (Sober, 1999), I claim that multiple realisation is commonplace, that it can be reductively explained, but that it requires a sui generis reductive explanatory strategy.
\end{abstract}

\section{Introduction}

An ever-increasing range of phenomena may be offered reductive explanations in terms of their lower-level constituents. As such, evidence for reductionism is growing. On the other hand, that some phenomena are multiply realised by different kinds of lower-level system poses a challenge to reductionism.

Multiple realisation (MR) implies that higher-level phenomena are independent of the lower level in the following sense: they are invariant with

*alexander.r.franklin@kcl.ac.uk 
respect to a swapping out of their lower-level constituents. ${ }^{1}$ This independence leads to a demand for explanation - how is it that multiple different kinds of system can exhibit the same phenomenon? The challenge to reductionism then depends on the claim that this demand cannot be met that the fact of multiple realisation cannot be reductively explained.

I take this challenge to be one which the reductionist ought to meet, and it's the purpose of this paper both to show that anti-reductionists are right not to be satisfied unless this challenge is answered, but that, with the right explanatory framework in hand, reductionists have the tools to vindicate their view. Consequently, I set out and defend a framework for providing reductive explanations of $\mathrm{MR} .^{2}$

This framework involves the identification of underlying commonalities in the various systems which instantiate the multiply realised phenomena. In addition, I claim that the features which distinguish such systems at the lower level must be shown not to make a difference to the multiply realised phenomena. This combination of identifying the commonalities with deriving that the distinguishing features don't make a difference guarantees the bottom-up prediction and explanation of multiple realisation. I illustrate this framework with a case study.

One of the advantages of this framework is that it makes clear the criteria that the reductionist has to fulfil in order to explain multiple realisation. This is important because the various accounts of reduction employed within this debate do not standardly require that the fact of multiple realisation be explained. As such, I argue that these more demanding constraints on reduction are appropriate if one wishes to address the anti-reductionists' concerns about MR. Note that, on this framework, reductionism does not imply eliminativism about higher-level kinds.

The remainder of the paper responds to potential objections. Some might claim (see e.g. (Polger and Shapiro, 2016)) that a phenomenon ought not to count as MR if it can be explained by the identification of commonalities - I argue that this amounts to the view that MR is inexplicable as a matter of conceptual necessity. For reasons developed below (see also e.g. (Aizawa, 2018)), I hold that this approach is mistaken. First, it's typical among both philosophers and scientists to accept that multiple realisation

\footnotetext{
${ }^{1}$ I use 'MR' to refer to 'multiple realisation', 'multiple realisability', 'multiply realised',

${ }^{2}$ While reductionism and MR were once presumed to be incompatible, (Bickle, 2020) shows that this view is increasingly rejected.
} 
is commonplace. If MR were, by necessity, inexplicable then a great many paradigm cases of MR would not count as such. This would suggest a re-labelling strategy that seems unlikely to catch on. Second, multiple realisation poses an explanandum which ought to be taken seriously, and which forms the basis of the anti-reductionist argument. Proponents of this objection would simply have to dismiss such a demand for explanation.

A second objection is that any adequate explanation of MR is necessarily anti-reductionist (see e.g. (Batterman, 2018) and (Morrison, 2012, 2014)). In countering these views, I claim that explanatory pluralism (see e.g. (Sober, 1999)) is insufficient. As such, I argue that an additional, sui generis reductive explanatory strategy ought to be added to the toolbox in order to rebut claims that MR is reductively inexplicable.

In $\S 2$ I set out what I take MR to involve, and how it might be explained. I develop this account with a simple case study from physics in $\S 3$. Then, in $\S 4$, I respond to various potential objections. In $\S 5$ I conclude.

\section{A Framework for Multiple Realisation}

\subsection{Definition}

A higher-level phenomenon $\mathrm{P}$ is multiply realised if and only if the same phenomenon $\mathrm{P}$ is realised in at least two different lower-level systems.

This definition is preliminary and requires clarification on two counts: what is meant by 'same phenomenon'? And what is meant by 'different lower-level systems'?

Following that clarification, in $\S 2.2$ I articulate what would be required for a reductive explanation of instances of MR which conform to this definition; and in $\S 4$ I relate this account of MR to others in the literature.

Phenomena are posited on the basis of experimental data and provide evidence for more general theories. Examples of phenomena include 'weak neutral currents, the decay of the proton, and chunking and recency effects in human memory' (Bogen and Woodward, 1988, p. 306). Phenomena are individuated by the scientific theory that describes them, thus we may say 
it's the same phenomenon if it is well described by the same theory in the same conditions. ${ }^{3}$

Importantly for compatibility with multiple realisability, phenomena are not individuated in terms of their realisers. ${ }^{4}$ Thus, as discussed below, an entity may be identified as an electrical conductor because it satisfies aspects of circuit theory, but it's not consequently possible to infer its specific constitution. A principal feature of systems instantiating MR is that they may be identified at multiple levels where higher-level identification does not commit us to any specific realisation.

What is meant by 'different lower-level systems'? Multiply realised phenomena are invariant with respect to certain changes in the lower-level system. I distinguish between two ways a system might be changed. ${ }^{5}$ On the one hand, consider varying the particular microstate of a system - i.e. changes which the system might actually undergo. On the other, consider varying the very nature and make-up of the system itself - these are counterlegal or imagined changes.

Phenomena which are invariant with respect to the first kind of change are called 'robust' - some phenomenon is robust if and only if it's invariant with respect to changes allowed by the laws or dynamics at the lower level. For example, most thermodynamic phenomena are robust across a wide range of different molecular arrangements, where the molecular laws allow the system to transition from one to another arrangement. ${ }^{6}$ The notion of changes allowed by the laws or dynamics is level-relative: when discussing the robustness or multiple realisation of a given phenomenon we (implicitly) specify a higher and lower level of description. Relative to such level-based descriptions a particular precision of description will be appropriate, and the allowed changes will be consequently defined.

Having set aside robustness, we can now refine the definition above:

\section{A higher-level phenomenon $\mathrm{P}$ is multiply realised if and only if}

\footnotetext{
${ }^{3} \mathrm{MR}$ is here defined in terms of (kinds of) phenomena, but MR may alternatively be defined in terms of kinds of entities. Nothing crucial to the argument relies on this choice: one may view electrical conductivity as a multiply realised phenomenon, or, alternatively, electrical conductor may be considered to be a kind of entity.

${ }^{4}$ See $\S 4$ for a discussion of various views which deny this.

${ }^{5}$ See (Hüttemann et al., 2015) for similar distinctions.

${ }^{6}$ Robustness is particularly relevant to characterising emergence, see e.g. (Butterfield, 2011; Franklin and Knox, 2018).
} 
the same phenomenon $\mathrm{P}$ is realised in at least two lower-level systems where the lower-level laws or dynamics forbid transitions between the states of those systems.

This distinction will be further exemplified in the next section where I claim that electrical conductivity is multiply realised by, say, lithium and potassium, because no realistic process can convert a strip of lithium into a strip of potassium. On the other hand, the electrical conductivity exhibited by strips of either metal will be robust for a range of different changes: the macroscopic physical properties will be the same even where constituent atoms have moved about.

The aim in making this distinction is to ensure that MR is not absolutely ubiquitous: every higher-level phenomenon is robust with respect to some changes, but MR phenomena are thought to prompt special explananda. ${ }^{7}$

Two further comments are relevant. First, non-fundamental levels are generically restricted both temporally and spatially; consequently, changes are nomologically impossible at some level if they happen so rarely that they fall outside the time-scale of that level. As such, a phenomenon may count as multiply realised by two different systems even where, on sufficiently long time-scales, some set of changes would lead to both systems' being well described by the same lower-level state. Thus, pressure in a sample of $\mathrm{CO}_{2}$ gas is robust because there are various different microstates which realise the same pressure, and transitions between those microstates are allowed by the lower-level laws. On the other hand, pressure in gases is multiply realised because both $\mathrm{CO}_{2}$ and $\mathrm{Ne}$ gases may realise the same pressure, but on timescales less than the age of the universe a sample of $\mathrm{CO}_{2}$ gas cannot change into one of $\mathrm{Ne}$ gas.

Second, this distinction will not define a division between robustness and MR which always squares with our intuitions. For example, certain colour changes in metals will be allowed by the lower-level laws or dynamics while others will not; thus, differently coloured metals are, in some instances, trivial but genuine examples of MR. Any way of excluding such boring cases would be arbitrary. As soon as we can explain a given instance of MR, it may count as less surprising, or even boring; but the requirement that MR be surprising would effectively undermine the interesting philosophical and scientific projects of explaining MR - such a stipulation would

\footnotetext{
${ }^{7}$ Alternatively, ubiquity could be avoided by adding further conditions; in $\S 4.1 \mathrm{I}$ argue that the conditions advocated by (Polger and Shapiro, 2016) are unattractive.
} 
beg the title question of the paper.

The distinction between MR and robustness is useful: it allows that, while there are genuine instances of MR in the world, not every higherlevel phenomenon is multiply realised; however, I do not claim that the distinction is profound. The distinction is nonetheless crucial to those who wish to claim both that MR is instantiated fairly widely, and that it is by definition incompatible with reduction. If those philosophers fail to make this distinction they will have a very difficult case to argue; if all robust phenomena count as multiply realised, and multiply realised phenomena are irreducible, then all robust phenomena will be classed as irreducible. Every phenomenon and every kind term identified in any science is robust with respect to some changes: no phenomenon only exists at, say, a single real-number-valued temperature. The failure of reduction would thus be absolutely generic. As far as I know, such a claim isn't made by advocates of the MR argument, and would leave their position rather more radical than is commonly acknowledged.

\subsection{Explanation and Reduction}

A further distinction is worth making at this stage between explanations that refer to MR phenomena, and explanations that tell us how MR is possible. One reason to take MR seriously is that the former kind of explanation is commonplace: many different scientific explanations refer to MR phenomena; that fact leaves it open whether or not explanations of MR are available. My project is non-eliminativist - as such I think it inadvisable to attempt to purge science of reference to MR phenomena. In other words, I do not purport to replace explanations which refer to MR phenomena, rather I aim to explain why such reference is successful.

With that distinction in hand, there are two more kinds of explanation. First, one may explain a given phenomenon in terms of each of its individual realisers - call this specificity explanation; second, one may explain that the phenomenon is multiply realised in terms of the common features shared by each realiser, and the details which make such common features sufficient for the occurrence of the phenomenon - call this commonality explanation. Specificity explanations do not explain the multiple realisation of the phenomenon, they rather focus on the individual behaviour in each lower-level system; commonality explanations, by contrast, do explain multiple realisation. For example, specificity explanations will ex- 
plain the particular conductivity of some metal, while commonality explanations will account for the multiple realisation of the conductivity.

This distinction corresponds to that employed by (Batterman, 2017, p. 570): "There are two kinds of "why-questions" that one might ask about a pattern of [multiply realised] behavior.

- Type I: One might ask why a particular instance of the pattern was realized.

- Type II: One might ask why the pattern itself exists across its different realizations'.

It's the second type of question that commonality explanations address - these demonstrate how multiple realisation comes about, not just why some particular phenomenon is instantiated in some particular system. ${ }^{8}$

In other words, one has merely restated the fact of multiple realisation if one demonstrates that system A exhibits phenomenon $\mathrm{P}$ and system $\mathrm{B}$ exhibits phenomenon P etc. As such, any system by system explanation will inevitably fail to address the type II explanandum. In order to explain the multiple realisation of phenomenon $\mathrm{P}$ it must be shown that all these systems display the same phenomenon in virtue of something which they share - that's why commonality explanations are required.

The way to offer a commonality explanation is, then, as follows: one explains the common behaviour by identifying an aspect or feature which is shared by the different systems, and one additionally demonstrates that the common features lead to the observed common behaviour in each case. While in some cases this second step may be left out, it is implicitly required.

When MR is instantiated and explicable, its realisers have both commonalities and heterogeneities; so, an explanation of MR must both identify the commonalities and demonstrate that the heterogeneities are irrelevant, in the sense that they do not make a difference to the MR phenomenon. That is, I claim that the only adequate explanations of MR are commonality explanations. As such, henceforth, all references to explanations of MR carry the implicit assumption that the explanation is a com-

\footnotetext{
${ }^{8}$ An explanation of this type will account for how the same phenomenon is realised in different 'ways' - see (Aizawa, 2018).
} 
monality explanation. ${ }^{9}$

Now we have a general model for how to explain MR, we ought to ask whether such explanations are reductive: is MR explained from the bottom up?

Insofar as reduction is possible, for any given phenomenon, one may come to understand why it is the way it is from the bottom up. That is, reductions dissolve the explanatory gaps which motivate anti-reductionists. As further discussed in $\S 4.2$, I think that standard accounts of reduction, which predict the phenomena for each realiser in turn, only address type I explananda. Type II explananda are those which motivate puzzlement about multiple realisation, and, as such, no reductive explanation of multiple realisation is adequate unless it addresses one of these.

An explanation of a multiply realised phenomenon is, thus, reductive insofar as it explains why that phenomenon is multiply realised with reference solely to lower-level details. The reductive constraint on explanation of MR amounts to the stipulation that the identified commonalities are lower-level commonalities, and that the irrelevance of the heterogeneities is secured by lower-level facts or processes.

Such reductive explanations need not be eliminativist in the sense that the MR phenomena are to be excluded from our ontology once they are explained. In fact, many reductive explanations will establish that the MR phenomenon should be included in our ontology. That's because by identifying the commonalities and the real, physical processes which secure the irrelevance of heterogeneities, we establish that the multiple realisation is not a pure artefact of our descriptive practices. We haven't simply imagined that the phenomenon is multiply realised - we can derive that fact from details of the realising systems. Reductive explanations of MR thus underwrite the value of the unified multiply realised description even while they allow us to understand, from the bottom up, why the multiple realisation obtains.

Note that, at various stages through the process of seeking to explain commonalities, we may acknowledge that the commonality is not in fact out there in the world; that, instead, it's illusory, or that it's a consequence of our epistemic limitations, or an artefact of our organisation of the world; we might also acknowledge that the MR is purely coincidental or a statis-

\footnotetext{
${ }^{9} \mathrm{It}^{\prime} \mathrm{s}$ further argued in $\S 4.2$ that, while explanatory pluralism is an attractive position, not all kinds of explanation can address all kinds of explananda.
} 
tical fluke. ${ }^{10}$ Where any of that happens the demands for explanation, and the prospects for anti-reductionism (in the case that reductive explanation fails) are correspondingly lessened - this overlaps with the debate over scientific realism, and, as such, will not be further discussed here. ${ }^{11}$

A reductive explanation of MR involves the identification of underlying common features and the demonstration, in lower-level terms, that such features are sufficient for the common behaviour. In the next section this framework for explaining MR is exemplified with a case study.

Note that different processes may be responsible in each realiser for securing the irrelevance of the heterogeneities. It's thus worth emphasising that, while the commonalities are in common, an account of each individual system is, in many cases, required in order to establish that the heterogeneities are irrelevant and, consequently, that the commonalities are sufficient for the MR phenomena to occur.

The upshot of this section is that MR is defined such that it is instantiated in the world and that reductive explanation is possible. I do not claim that all cases of MR can be offered reductive explanations; in my view, that's an empirically sensitive question, and reduction is hard! The advantage of my framework is that that question can be addressed, and, especially in mature sciences, reductive explanations of MR can be provided.

\section{A Case Study}

The reductive strategy outlined above is, in this section, cashed out in terms of a prototype reductive explanation of multiple realisation.

Consider the phenomenon that various different metals conduct electricity. ${ }^{12}$ For our purposes, we may restrict attention to the Alkali (group I) metals; although electrical conductivity is, of course, multiply realised by a far wider class of materials, the reductive story is much simpler if we stick

\footnotetext{
${ }^{10}$ Some statistical flukes might be selected for - this would lead to MR which could not be reductively explained in a way that should not trouble the reductionist. However, the process of selection might pose its own challenges to reduction.

${ }^{11} \mathrm{It}^{\prime}$ 's worth emphasising that the question here is not whether we should be metaphysical realists about the universal which putatively underlies a given common feature; rather we are interested in whether or not there is any stable way of identifying the putative commonality such that it doesn't depend on the way we happen to do science.

${ }^{12}$ This example is in part inspired by that in (Aizawa, 2013).
} 
to this restricted class, and the philosophical moral generalises.

It's worth emphasising that this example is fairly naturalistic: we needn't think of electrical conductivity in terms of human interactions it manifests, for example, when lightning strikes. If one still has reservations that the example is unacceptably ad hoc or anthropocentric, (Franklin, 2019) demonstrates that a similar reductive approach may be applied to the universality of critical phenomena, which, as briefly discussed in $\S 4.2$, has been touted by some as a paradigm instance of irreducible MR.

Moreover, the phenomenon fits my definition: first, the phenomenon of conduction of electricity in lithium is identical to the phenomenon of conduction of electricity in all the other group I metals. As noted above, the identity of phenomena depends on the higher-level scientific theory. Solid state physics, see e.g. (Kantorovich, 2004), uses the same formalism to describe the conductivity of all such metals. At a greater level of abstraction, circuit theory also uses the same functional relationships to describe this phenomenon independently of which particular metal realises the conductivity. Note that neither solid state physics nor circuit theory are parts of fundamental physics!

Second, the realisers of electrical conductivity are different. Group I metals all have many behaviours in common, but do have clear differences: for example they have different resistivity, different melting and boiling points, different densities, and they have differently coloured flames. ${ }^{13}$ Although fission and fusion may occur in specialised circumstances at an atomic level, a piece of lithium will not transform into a piece of sodium within a time-scale of the order of the age of the universe. ${ }^{14}$ Despite their sharing a variety of physical properties, there is no non-question-begging way of saying that lithium is identical to, say, sodium.

Their electrical conductivity is the multiply realised phenomenon which serves as my case study. If one prefers to think of MR in terms of kinds, then this could be substituted for the claim that electrical conductor is multiply realised by these different metals.

Can the fact that electrical conductivity is multiply realised be reductively explained? Following the framework outlined above, such explana-

\footnotetext{
${ }^{13}$ Lithium, sodium, potassium, rubidium and caesium have red, yellow, violet, yellow violet and blue flames respectively; see (Dye and Tepper, 2018).

${ }^{14}$ Their difference is thus a consequence of the fact that the level on which lithium is identified is temporally restricted.
} 
tion requires the identification of both the commonalities among the realisers and the features which make the heterogeneities irrelevant to the shared behaviour, all in lower-level terms.

Two features of group I metals make them such good conductors: the fact that they have a single electron in their outer shell, and the arrangement of the repeated unit cells of the metal's lattice structure.

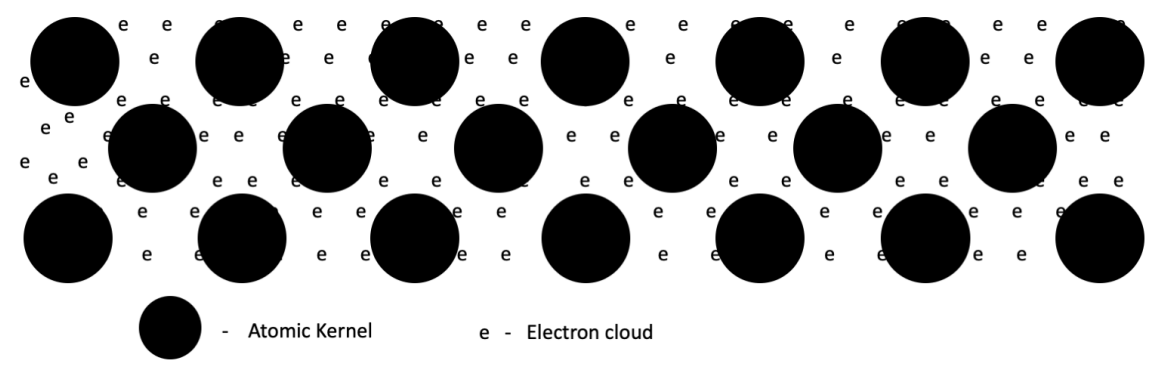

Figure 1: A representation of the distribution of electrons in a metal. This is not to scale.

Group I metals all have a free electron in their outer shell. This electron may, with little energetic cost, dissociate from its atom. As displayed in figure 1 , such electrons form a cloud which is distributed among the atoms in the lattice. The crucial feature which allows for conductivity is that the quantum wavefunction which represents the state of each electron will then be delocalised and spread across the whole material. The Pauli exclusion principle precludes such electrons from inhabiting the same state, as such they are effectively non-interacting in the delocalised cloud, which is consequently well described as a freely moving gas.

The regular lattice structure in figure 1 means that there is no net force acting on the cloud. This has the consequence that, when an electric field is applied, the electron gas may freely travel in the direction of the field lines and form an electric current. In brief: dissociated electrons form quantum mechanical waves in periodic potentials - that's what allows electrical conductivity to be exhibited in multiple different metals.

The commonality among all the metals is that their atoms all have the same number of electrons in their outer shell and that their atoms are arranged into similar lattice structures. These are, of course, lower-level commonalities. Although conductivity is identifiable at the higher level, the features which lead to common electrical conductivity in various systems can be specified in lower-level terms. In addition, the electric current can 
be identified in lower-level terms as a net flow of electrons.

The remaining feature to be explained is that the differences between the metals - the heterogeneities - are irrelevant to the phenomenon of interest. Only if that can be established may we explain how the common features are sufficient for the common behaviour. The irrelevance of heterogeneities may, however, also be explained in lower-level terms. There are two salient features which distinguish group I metals at the atomic scale. First, each metal has a different number of electrons - that difference is irrelevant due to the stability of the filled shells. At low temperatures, electrons in inner shells are not readily excited and their different numbers are irrelevant. Although at higher temperatures the system will be more energetic and the inner electrons may become relevant, this does not undermine the explanation - it is a generic feature of multiple realisation that the common behaviour is only exhibited in restricted contexts.

The second way in which group I metals differ from each other lies in their different proton and neutron numbers. However, the different constitutions of the nuclei are irrelevant since they all compose lattice structures. As noted above, this structure ensures a periodic potential which allows the electron gas to flow freely throughout the metal. The periodic potential of the lattice can be explained in terms of the underlying atomic bonding which leads to the lattice formation.

While the heterogeneities are, thus, shown to be irrelevant to the metals' conductivity, this does not imply that the commonalities (singly) realise the conductivity. It's physically nonsensical to consider a metal or its conductivity in the absence of its protons and neutrons! This point is discussed further in $\S 4$.

It's important to bear in mind that, while this simplified case study involves a similar account of the irrelevance of heterogeneities for each realiser, in many contexts such stories will diverge - so long as the different systems have different underlying properties and these are irrelevant, the process by which they are irrelevant may differ for each system. Even in this case study, the processes which secure irrelevance of heterogeneities may differ between materials: I noted that the different nuclear constitutions are screened off by the fact that the electrons interact with a periodic potential, but the fact that there's a periodic potential depends on the lattice structure, which may differ somewhat among different metals. This is a mild difference, but it illustrates the broader point that part of explaining 
MR involves taking account of the peculiarities of each realiser.

In sum, we may adduce, in lower-level terms: first, the commonalities alkali metals all have a single outer-shell electron and similar atomic lattice structures; and second, the irrelevance of the heterogeneities which follows from the stability of the inner shells and the periodicity of the lattice structure.

\section{Objections and Responses}

\subsection{MR is Essentially Inexplicable}

Some might object to my claim that MR can be reductively explained because they hold MR to be inexplicable by commonality explanations as a matter of conceptual necessity. As argued above and further developed in $\S 4.2$, commonality explanations are the only way to provide explanations of multiple realisation.

In this section I argue that it is a mistake to define MR as essentially inexplicable: the issues at stake in this paper ought to be settled by empirical investigation - facts about the world make it such that particular cases of MR are explicable and reducible, or not.

I resist the conflation of MR with inexplicability and irreducibility and argue that whether or not a phenomenon is MR is, at least to some extent, independent of whether or not it can be reductively explained. A major reason to maintain this distinction is that we may identify and discuss MR phenomena without thereby committing ourselves to an account of reductive explanation. As such, by resisting the conflation we conserve a term for the interesting fact that various phenomena exhibit the confluence of commonality and heterogeneity which corresponds to MR. Whatever one's standards for reduction, it should be acknowledged that instances of MR still pose interesting and important explananda for scientific research.

Maintaining the distinction between inexplicability and MR allows reductionists and anti-reductionists alike to acknowledge that some phenomena are multiply realised, that these pose a challenge for reductive explanation, but that that challenge may not be insuperable. Whether or not the challenge can, in fact, be overcome is then to be decided on a case by case basis rather than in principle. 
Polger and Shapiro (see their (2016), (Shapiro, 2018), and references therein) have done a great deal of work to demonstrate that, for various putative instances of MR, the realisers in fact share common causal structure. There is, thus, a sense in which their project can be construed as providing reductive explanations of MR. However, they are committed to the conflation just outlined. That is, they argue that putative cases of MR ought not to count as such insofar as they can be reductively explained by commonality explanations.

According to (Shapiro, 2000, p. 646):

two realizations of a kind $T$ are in fact different kinds of realizations of $T$ only when they differ in their causally relevant properties, that is, the properties by which they contribute to the capacity, purpose, goal, and the like that serves to individuate $T$ as the kind that it is.

Shapiro goes on to argue that MR is in fact rather rare, because in many putative instances of MR the two realisations don't differ with respect to causally relevant properties, they rather differ with respect to their causally irrelevant properties. For example, given the analysis in the previous section, his account implies that one ought not to say that the kind electrical conductor is multiply realised simply in virtue of the fact that both lithium and potassium conduct electricity. This is because the features which distinguish lithium from potassium might be claimed to be causally irrelevant to individuation qua electrical conductor.

This view is defended by (Polger and Shapiro, 2016) who consider a range of neurological case studies that have been described as instances of MR. They suggest that in almost all such cases the causal process which gives rise to the putatively multiply realised kind is identical. ${ }^{15}$ For them, in order to qualify as MR, the relevant causal structure must be different.

While Polger and Shapiro have done significant and important work in bringing empirical considerations to bear on discussions of MR, it's unfortunate that they are committed to this account of MR. Not only does it make MR essentially inexplicable by commonality explanations (because

\footnotetext{
${ }^{15}$ While they (ibid., p. 73) accept that genuine multiple realisation is commonly found among artefactual kinds, they note that the interesting philosophy of science questions presuppose naturalistic multiple realisation.
} 
such explanations would mean it no longer counted as MR), but it also undermines an important scientific project: that of accounting for how it is that there are so many instances of higher-level phenomena realised by multiple systems distinct at the lower level. ${ }^{16}$ Important work in countering this aspect of Polger and Shapiro's project has been carried out by Aizawa and Gillett (see e.g. (Aizawa and Gillett, 2009)), who argue that multiple realisation is pervasive in the natural world, and provide a partial explanation of that fact for some biological systems. In addition, (Aizawa, 2013) also offers an explanation of the pervasiveness of MR in some physical systems.

Thus, my framework in $\S 2.2$ may be used to interpret aspects of Gillett and Aizawa's work as offering commonality explanations of MR. ${ }^{17} \mathrm{How}-$ ever, their account is not explicitly targeted at reductive explanations of MR, and they do not discuss the type II explanandum. Consequently, their account is, in general, insufficient to address the anti-reductionists' worries about MR, though that is not their explicit target. ${ }^{18}$

In this paper, I show how a general framework for reductive explanation is compatible with MR, and that, once one recognises this fact, one can engage with the interesting project of identifying cases of MR, and assessing whether or not these can be reductively explained. Some of the explanations offered by Aizawa and Gillett fit into this framework and may be used to offer reductive explanations of certain instances of MR phenomena.

Polger and Shapiro differ from me, and from Aizawa and Gillett, in their account of multiple realisation. In my opinion, they err in claiming that once common causal structure has been identified, the phenomena in question should no longer count as multiply realised. If one were to accept Polger and Shapiro's analysis, one would be committed to categorising putative cases of MR as either inexplicable, or not multiply realised. By resisting the conflation, the category of MR-but-explicable is maintained and the interesting scientific explanations of how multiple realisation comes about are viable. In other words: once we accept that MR is reductively explicable in principle, we can engage in the framework laid out above which asks whether, in practice, particular instances of MR can be reductively ex-

\footnotetext{
${ }^{16}$ For a related argument that this account does not aid the reductionist project, see (Bickle, 2010).

${ }^{17}$ An additional aspect of their work focusses on the metaphysics of realisation - see (Gillett, 2003); I don't advocate any particular stance in that debate in this paper.

${ }^{18}$ The framework for reduction in (Gillett, 2016) does not consider type II explananda.
} 
plained.

On the account defended here, it's a discovered fact about the world that there are many phenomena which are identified independently of their realisation and are instantiated in multiple different kinds of system. Failing to acknowledge this as an explanandum would be to sidestep the substantial challenge to reduction which MR (or putative MR) poses; this line of reasoning is further defended in $\S 4.2$.

On my view, multiple realisation is fairly commonplace, but can be explained. Polger and Shapiro's view is that it's much rarer but can't be explained. In (Polger and Shapiro, 2016, p. 39), they defend their view by claiming that a more liberal view 'entails an undesirable profligacy of distinct realizations for every kind, and undermines the significance of realization within debates over the autonomy of the special sciences'. While I agree that just any lower-level variation is insufficient for MR, I think that the MR-robustness distinction drawn above in $\S 2$ rules out 'undesirable profligacy'. On the other hand, regarding autonomy, I'd argue that the autonomy of the special sciences is, in fact, closely related to multiple realisation as I define it: as Polger and Shapiro acknowledge (ibid., chapter 10.4), a system has some claim to autonomy if it provides for prediction and explanation at the higher level. Insofar as such autonomy can be explained, the threat to reduction is mitigated, but the debate over such cases is only confused by claiming that autonomy, or indeed MR, is rare but mysterious.

Lastly, even if Polger and Shapiro's approach is taken to provide an explanation of MR, their explanatory strategy is somewhat lacking. That's because, while they rightly emphasise the importance of demonstrating what's in common between the realisers, ${ }^{19}$ they do not require that we also demonstrate that the heterogeneous features are irrelevant; and it is precisely this extra step that is required to dispel the apparent mystery that different systems exhibit common behaviour.

It's the very fact that different systems act similarly which is so remarkable - that's what motivates (Fodor, 1974) in his discussion of the multiple realisation of currency and (Putnam, 1975) in his discussion of the multiple realisation of square and round pegs. That there is MR is a consequence of the fact that genuinely different systems do, in some circumstances, ex-

\footnotetext{
${ }^{19}$ Though their emphasis on common causal structure will be unattractive to those who question the applicability of causal vocabulary to the physical sciences; see e.g. (Price and Corry, 2007).
} 
hibit identical phenomena notwithstanding their differences. MR is thus explained by showing both that such systems have features in common, and that, in those circumstances, their differences are irrelevant. By failing to offer explanations of this form, those who conflate inexplicability with MR cannot adequately respond to the anti-reductionist's challenge.

In part, my commitment to the distinction between MR and inexplicability stems from analysis of case studies from physics. In this context, we have phenomena which are quantitatively identical across a range of different systems; as such, there is good reason to think that multiple realisation is not an artefact of our classificatory practices. Examination of cases from physics also helps establish that reductive explanations of multiple realisation are the subject of active scientific inquiry.

The examination of case studies from the physical sciences is particularly helpful in these fraught philosophical discussions. Cases drawn from the biological sciences are far more complex and, consequently, reductive explanation is harder to come by. Therefore, in such contexts it's difficult to distinguish whether the source of apparent irreducibility is multiple realisation, or some other feature of the target system. Physics examples are, in general, more susceptible to reductive explanation, and the MR challenge can, thus, be assessed independently of confounding factors. However, the discussion in the philosophy of physics literature has led to a different objection to an approach like my own.

\subsection{Commonality Explanations of MR are Anti-reductionist}

I claimed in $\S 2.2$ that specificity explanations of MR phenomena are inadequate to address the MR explanandum. Once we have a reductive explanation for why lithium is a good electrical conductor, and why sodium is a good electrical conductor we still have an explanatory demand: what common feature of group I metals leads to the phenomenon that they all conduct electricity? Where the same phenomenon is observed in all these different systems we have multiple realisation and thus we have an $e x$ planandum for which the reductionist ought to be able to provide a reductive explanation.

Some might argue that no reductive explanation could address such explananda. For example, Batterman's view (e.g. $(2000,2018)$ ) is that there is $\mathrm{MR}$, and that it can be explained, but that MR can only be explained 
anti-reductionistically. ${ }^{20}$ As such, he denies the potential for the kind of reductive explanatory strategy outlined above. An important insight due to Batterman, on which I build in this paper, is that the MR explanandum requires a sui generis explanatory strategy. While I have argued that carrying out such a strategy can provide evidence for reduction, and Batterman disagrees, I follow him in claiming that once one acknowledges that there is MR in the world, a novel explanandum follows.

It's particularly worth highlighting this set of claims due to Batterman because they serve to undermine the explanatory pluralism advocated by (Sober, 1999):

Generality is one virtue that an explanation can have, but a distinct - and competing - virtue is depth, and it is on this dimension that lower-level explanations often score better than higher-level explanations. The reductionist claim that lowerlevel explanations are always better and the anti-reductionist claim that they are always worse are both mistaken.

[(Sober, 1999, p. 560), original emphasis]

Sober argues that multiple realisation need not trouble the reductionist. He does so by observing that different kinds of explanation are useful or applicable to different ends. He observes that adding content to an explanation does not stop its being an explanation and that reductions which offer bottom-up explanations - will generally be of interest even if the higher-level explanations are adequate in some contexts.

Sober's arguments are well-taken: it's certainly the case that proportionate higher-level explanations are often superior qua explanations of higher-level explananda. However, not just any kind of explanation is adequate to explain MR. Given that Sober is talking about multiple realisation and explanatory approaches thereto, it seems fair to say that he has missed the anti-reductionist's point. This observation is emphasised by (Batterman, 2018, 2000), who identifies an explanandum which is missed by many reductionists: ${ }^{21}$

\footnotetext{
${ }^{20}$ (Batterman, 2000, p. 134): '[m]y position is unusual because it asserts the possibility of providing physical explanations for why certain special science properties are multiply realized without also providing a reduction'.

${ }^{21}$ Note that (Morrison, 2012, pp. 164-165) makes a similar point - she argues that what I call specificity explanations are inadequate to explaining instances of MR and that some
} 
MR: How can systems that are heterogeneous at some (typically) micro-scale exhibit the same pattern of behavior at the macro-scale?

$\ldots$

if one thinks (MR) is a legitimate scientific question, one needs to consider different explanatory strategies. The renormalization group and the theory of homogenization are just such strategies. They are inherently multi-scale. They are not bottom-up derivational explanations.

[(Batterman, 2018, pp. 4, 14-15)]

I agree with Batterman that standard reductionist approaches miss the MR explananda. ${ }^{22}$ The question labelled MR prompts us to look at the distinct realisers of multiply realised phenomena and ask why all of these different underlying systems realise the same higher-level phenomenon. Batterman counters Sober's explanatory pluralism by arguing that, in certain cases, answers to MR are incompatible with reduction. He claims that universality is multiple realisation and that the full explanation of universality necessarily proceeds at the higher level. Batterman's contention is that lower-level and higher-level explanations are not equally adequate for understanding cases of MR. In fact, specifically for certain such cases the higher-level (or multi-level) explanations are the only ones which are able to address the principal explanandum. ${ }^{23}$

While I disagree with Batterman's anti-reductionist conclusion, I accept his more general assertion: that it's not good enough simply to say, as Sober does, that different explanations are good for different ends. The different descriptions and explanations of electrical conductivity - some in terms of abstracted circuit theory, and others in terms of the microscopic details of each metal - do not lead to an answer to MR. And it is that question which really provides the motivation for the anti-reductionist. Sober's pluralism is inadequate to refute the assertion that multiple realisation is inexplicable from the bottom up.

top-down (anti-reductionist) constraints are necessary. (Ross, 2020) makes a similar point with a focus on causal explanation.

${ }^{22}$ See e.g. (Kim, 2005).

${ }^{23}$ Although Batterman thinks that universality can be offered an explanation, he contends that this explanation is unavailable from the bottom up due to its appeal to renormalisation group (RG) methods; his technical argument is criticised in e.g. (Saatsi and Reutlinger, 2018; Franklin, 2019). 
I have argued in this paper that MR may nonetheless be answered reductively. That is, I claim that, if we adduce the commonalities among lower-level realisers and identify the lower-level processes which make the heterogeneities irrelevant to the common behaviour, then we have provided a reductive explanation of MR. As such, the anti-reductionist's motivations may be addressed, and the compatibility of MR with reduction may be established. MR poses an explanandum which requires a sui generis explanation; it's just that reductive explanations of MR are available.

\section{Conclusion}

Multiple realisation arguments have been taken by many to settle the case against reduction. And yet there's a fair bit of ambiguity over the structure of the argument. One strand of the debate observes that, given multiple realisation, the higher-level facts do not determinately pick out unique lowerlevel states. However, rather than an argument, this is more a restatement of multiple realisation, and it is hardly surprising: everyone should accept that higher levels are more coarse-grained and less determinate than lower levels.

A better argument notes that multiple realisation corresponds to invariance with respect to a swapping out of the lower-level constituents. The consequent independence poses an explanatory challenge - how come a common higher-level description is available despite lower-level heterogeneity? This challenge is one that the reductionist ought to meet, and, in this paper I showed that this challenge may be answered.

It's worth considering once again the response of those philosophers who'd define MR such that, in principle, it's not reductively explicable. First, they might argue that the kind of commonality explanation discussed here is insufficient for reduction. As such, they might accept that MR can be explained in the sense articulated above, but that reduction is ruled out. Importantly, I think that MR is, in general, a sign that we should think the higher level ineliminable: that, in most cases, MR phenomena should be included in our ontological inventory. As such, I have no quarrel with those who take MR to be incompatible with eliminativist reduction. I have merely argued that this does not imply that MR is mysterious or inexplicable from the bottom up. 
Second, they might claim that, while the framework developed here does lead to greater understanding of various worldly phenomena in many contexts, such phenomena do not count as MR. MR would thus be rather rare, but wherever it was in fact instantiated, it would be inexplicable. I think that by conflating multiple realisation with inexplicability, such an account would lose the important category of MR-but-reductively-explicable. It's far better to define MR such that it poses an explanatory challenge which can, in some though not necessarily all cases, be addressed.

In this paper, I claimed that multiple realisation is found whenever the same phenomenon is realised by multiple different systems. I went on to argue that it is best to view the MR argument against reduction as posing a novel explanandum. MR requires new explanations in addition to those provided by traditional reductive approaches; the availability of such explanations must be assessed on a case by case basis. Thus, MR does raise a problem for reduction, and it's an empirically sensitive matter whether or not reduction can withstand the MR argument.

\section{Acknowledgements}

I'm very grateful to Ken Aizawa, Donald Franklin, Eleanor Knox, Jess Leech, several anonymous referees, attendees of the MetaScience Work in Progress group, attendees and co-symposiasts of the Multiple Realisability Symposium at BSPS 2019, and attendees of various graduate research seminars at King's College London for many helpful questions and comments that have led to the substantial improvement of this paper. This work was partly funded by ERC Horizons 2020 grant no. 771509 through the Metaphysical Unity of Science (MetaScience) project.

\section{References}

Kenneth Aizawa. Multiple realization by compensatory differences. European Journal for Philosophy of Science, 3:69-86, 2013. doi: 10.1007/ s13194-012-0058-6.

Kenneth Aizawa. Multiple realization and multiple "ways" of realization: A progress report. Studies in History and Philosophy of Science Part A, 68: 3-9, 2018. 
Kenneth Aizawa and Carl Gillett. Levels, individual variaty, and massive multiple realization in neurobiology. In John Bickle, editor, The Oxford Handbook of Philosophy and Neuroscience. Oxford University Press, 2009. doi: 10.1093/oxfordhb/9780195304787.003.0023.

Robert W. Batterman. Multiple realizability and universality. The British Journal for the Philosophy of Science, 51(1):115-145, 2000.

Robert W. Batterman. Philosophical implications of kadanoff's work on the renormalization group. Journal of Statistical Physics, 167(3-4):559-574, 2017.

Robert W. Batterman. Autonomy of theories: An explanatory problem. Noûs, 52(4):858-873, 2018. doi: 10.1111/nous.12191.

John Bickle. Has the last decade of challenges to the multiple realization argument provided aid and comfort to psychoneural reductionists? Synthese, 177(2):247-260, 2010.

John Bickle. Multiple realizability. In Edward N. Zalta, editor, The Stanford Encyclopedia of Philosophy. Metaphysics Research Lab, Stanford University, summer 2020 edition, 2020.

James Bogen and James Woodward. Saving the phenomena. The Philosophical Review, 97(3):303-352, 1988.

Jeremy Butterfield. Emergence, reduction and supervenience: A varied landscape. Foundations of Physics, 41(6):920-959, Jun 2011. doi: 10.1007/ s10701-011-9549-0.

James L. Dye and Frederick Tepper. Alkali metal. In Encyclopædia Britannica. June 2018. URL britannica.com/science/alkali-metal.

Jerry A. Fodor. Special sciences (or: The disunity of science as a working hypothesis). Synthese, 28(2):97-115, 1974.

Alexander Franklin. Universality reduced. Philosophy of Science, 86(5), 2019. doi: $10.1086 / 705473$.

Alexander Franklin and Eleanor Knox. Emergence without limits: The case of phonons. Studies In History and Philosophy of Modern Physics, 64:68-78, 2018. doi: 10.1016/j.shpsb.2018.06.001.

Carl Gillett. The metaphysics of realization, multiple realizability, and the special sciences. The Journal of Philosophy, 100(11):591-603, 2003. 
Carl Gillett. Reduction and emergence in science and philosophy. Cambridge University Press, 2016.

Andreas Hüttemann, Reimer Kühn, and Orestis Terzidis. Stability, emergence and part-whole reduction. In Margaret Morrison and Brigitte Falkenburg, editors, Why More is Different: Philosophical Issues in Condensed Matter Physics and Complex Systems, chapter 10. Springer, 2015.

Lev Kantorovich. Quantum theory of the solid state: an introduction, volume 136. Springer Science \& Business Media, 2004.

Jaegwon Kim. Physicalism, or something near enough. Princeton University Press, 2005.

Jennifer A. Lewis. Department of materials science and engineering university of illinois (uiuc). Materials Science and Technology Module Web Site, 1995. URL http://matsel.matse.illinois.edu/metals/prin. html.

Margaret Morrison. Emergent physics and micro-ontology. Philosophy of Science, 79(1):141-166, 2012. doi: 10.1086/663240.

Margaret Morrison. Complex systems and renormalization group explanations. Philosophy of Science, 81(5):1144-1156, 2014. doi: 10.1086/677904.

Thomas W. Polger and Lawrence A. Shapiro. The Multiple Realization Book. Oxford University Press, 2016.

Huw Price and Richard Corry, editors. Causation, Physics, and the Constitution of Reality: Russell's Republic Revisited. Oxford University Press, 2007.

Hilary Putnam. Philosophy and our mental life. In Mind, Language and Reality: Philosophical Papers, Volume 2, pages 291-303. Cambridge University Press, 1975.

Lauren N Ross. Multiple realizability from a causal perspective. Philosophy of Science, 2020. doi: 10.1086/709732.

Juha Saatsi and Alexander Reutlinger. Taking reductionism to the limit: How to rebut the antireductionist argument from infinite limits. Philosophy of Science, 85(3):455-482, 2018. doi: 10.1086/697735.

Lawrence A. Shapiro. Multiple realizations. The Journal of Philosophy, 97 (12):635-654, 2000. 
Lawrence A. Shapiro. Reduction redux. Studies in History and Philosophy of Science Part A, 68:10-19, 2018.

Elliott Sober. The multiple realizability argument against reductionism. Philosophy of Science, pages 542-564, 1999. 